\title{
Didier Boisson, Consciences en liberté ? Itinéraires d'ecclésiastiques convertis au protestantisme
} (1631-1760)

Paris, Honoré Champion, 2009, 778 p.

Daniel Vidal

\section{CpenEdition \\ Journals}

Édition électronique

URL : http://journals.openedition.org/assr/21522

DOI : $10.4000 /$ assr.21522

ISSN : $1777-5825$

Éditeur

Éditions de l'EHESS

Édition imprimée

Date de publication : 31 décembre 2009

ISBN : 978-2-7132-2218-4

ISSN : 0335-5985

Référence électronique

Daniel Vidal, « Didier Boisson, Consciences en liberté? Itinéraires d'ecclésiastiques convertis au protestantisme (1631-1760)», Archives de sciences sociales des religions [En ligne], 148 | octobredécembre 2009, document 148-24, mis en ligne le 15 novembre 2012, consulté le 21 septembre 2020. URL : http://journals.openedition.org/assr/21522 ; DOI : https://doi.org/10.4000/assr.21522

Ce document a été généré automatiquement le 21 septembre 2020.

(C) Archives de sciences sociales des religions 


\section{Didier Boisson, Consciences en liberté? Itinéraires d'ecclésiastiques convertis au protestantisme (1631-1760)}

Paris, Honoré Champion, 2009, 778 p.

Daniel Vidal

\section{RÉFÉRENCE}

Didier Boisson, Consciences en liberté ? Itinéraires d'ecclésiastiques convertis au protestantisme (1631-1760, Paris, Honoré Champion, 2009, 778 p.

$148-24$

De l'allégeance militante à l'Église « romaine ", à l'adhésion aux Églises calvinistes en France ou Genève et Londres, les chemins de la conversion sont toujours complexes, tissés de raisons intérieures parfois avouées, le plus souvent demeurées dans l'ombre des consciences repenties. Définir les éléments qui décident, aux XVII et $\mathrm{XVIII}^{\mathrm{e}}$ siècles, de cette mutation radicale, suppose que l'on multiplie les hypothèses afin de pénétrer plus avant dans le déchiffrement des itinéraires «prosélytes». Sans doute quelque obligation éthique, quelque raison théologique, ou quelque rencontre avec un initiateur convaincant, ou des lectures à la marge de l'institution romaine, peuvent-elles être sollicitées, selon les récits de convertis, pour que l'on puisse comprendre le sursaut d'une conscience qui se découvre « libre » et ose transiter de l'une à l'autre Église. C'est tout l'extrême intérêt de la recherche de Didier Boisson: comment penser le détachement, soudain ou lentement mûri, d'ecclésiastiques rattachés aux valeurs et rites de Rome, pour adhérer à la "nouvelle religion", cette "hérésie " dès le début combattue par leur propre Église, et quelle extension connut ce mouvement de conversion? 
2 L'auteur rappelle la double signification de l'acte de conversion : un retour sur soi, une interrogation sur les fondements même d'un engagement cultuel ; mais aussi bien, un exact repentir, après que fussent devenus manifestes les errements antérieurs, et, en quelque sorte, une véritable renaissance. Les récits, peu nombreux, mais précieux, des raisons invoquées par quelques convertis, pour obéir parfois à un schéma convenu, ne distinguent pas aussi abruptement cette quête personnelle et cette repentance, cette descente au cœur d'une identité fervente, et cette mise au net d'une foi nouvelle enfin révélée. Non plus qu'une temporalité propre à la décision de faire conversion, reliant ainsi les deux modèles - paulinien (évènement inattendu), et augustinien (long parcours et action réfléchie). Le rejet de ce qui est alors tenu pour erreurs de l'Église catholique est le plus souvent indissociable de la recherche passionnée d'un christianisme tel que pratiqué en son " origine ». Si bien que la mise en question de soimême en tant que croyant rejoint la nécessité de penser seule véritable, l'Église en sa primitive passion. En ce sens, Calvin, dont on peut considérer la conversion comme un modèle combinant des tensions multiples, témoigne de l'enchevêtrement des causes : terreau humaniste érasmien, rencontre d'un initiateur à la doctrine naissante, parcours de doute et de réflexion, etc. La conversion? «Soudaine et subie, immédiate et passive ", mais issue du plus profond d'une quête personnelle.

3 L'échantillon de quatre cent cinquante conversions d'ecclésiastiques, que D. Boisson a pu constituer à partir des Archives d'État de Genève (consistoire, pasteurs, Chambre des prosélytes) et de la Banque de données informatiques sur le refuge huguenot, atteste sur la longue durée (1630-1760 - soit de la lente érosion du protestantisme en France, jusqu'à sa difficile restauration civile et civique), de la permanence des modalités par quoi se définit cet acte radical de rupture. Les convertis viennent en majorité des ordres mendiants, notamment franciscains, mais des capucins, aussi bien, et des carmes. Si l'un d'entre eux, Jean de Labadie, jésuite puis carme, vient à la Réforme en 1650, afin, comme il en va de tout converti, de retrouver une "pureté " que l'Église catholique n'observe plus, il serait intéressant de comparer cette conversion à celle, en sens inverse, de Benoît de Canfield, anglican passé en France, à la fin du XVI e siècle, et entré en l'Ordre des capucins pour y trouver la "pureté » et la rigueur que son Église d'origine avait abandonnées. Au-delà de l'anecdote, cela fait problème : la quête de la pureté, de la rigueur, la tentation/tentative de faire renaître de ses cendres une Église en tous points fidèle aux impératifs du Texte profané, suppose un travail au centre de la spiritualité de chacun. Est-ce un hasard si les ordres contemplatifs fournissent plus d'un converti? Les jésuites, de leur côté, au début des années 1630/1640, connaissent quelque passion mystique. En la province d'Aquitaine le groupe des " petits saints » qui se constitue informellement en cette région, - et dont Joseph Surin, J.-J. Baiole, J. du Tertre, sont les exemples les plus connus -, favorise les premières effervescences mystiques de Labadie, qui vient à la Réforme au milieu du $\mathrm{XVII}^{\mathrm{e}}$ siècle. Il en va de même de plus d'un ecclésiastique, dont tel itinéraire, pour singulier qu'il soit, représente aussi bien une démarche partagée. De la passion mystique à l'esprit de Réforme, si je peux me permettre de reprendre le sous-titre de mon ouvrage sur Labadie, il n'est pas de frontière absolue, bien au contraire. François Clouet, capucin régulier, se convertit en 1639, exerce son ministère aux ProvincesUnies, et retourne au catholicisme huit ans plus tard. Yves de Vallone, prêtre, chanoine régulier de Sainte-Geneviève, est agréé comme ministre réformé, et de ce passage d'une fonction prédicatrice, à cette autre, exactement similaire, il est possible, sans nier la rupture que cette transition suppose, de faire apparaitre des continuités 
insoupçonnées. De ce point de vue, les sermons prêchés par Labadie à Montauban, Genève, Middelbourg, Amsterdam, s'inscrivent dans le droit-fil de ses premières méditations chez les jésuites aquitains.

4 La conversion n'apparaît pas alors, ou pas entièrement, comme une divine surprise. La surprise tiendrait plutôt en une certaine continuité dans la quête éperdue d'un modèle d'Église, toute foi assumée en ses variations, et toute incertitude. Elle ne serait que l'ultime accomplissement d'une exigence radicale concernant l'acte de croyance, et son assomption. Elle est précédée souvent d'une véritable «errance » institutionnelle, que D. Boisson souligne chez Clouet, Vallone, Labadie, pour s'en tenir aux plus exemplaires des cas. Labadie fut jésuite, jansénisant, carme, proche des «illuminés » de Picardie, etc. Clouet, capucin, est "ballotté entre plusieurs couvents de l'ordre", Yves de Vallone, génovéfain, parvient difficilement à s'intégrer dans les établissements où il est affecté. D. Boisson multiplie les exemples d'ecclésiastiques en errance. Mais cette difficulté de stabilisation s'accompagne, ou témoigne, d'une hésitation dans l'ordre de la spiritualité. On sait combien a pu peser chez Labadie, dans le passage à la Réforme, ce « nomadisme » que Michel de Certeau avait très précisément identifié, et qui ne cessera de qualifier ses tribulations en toutes places de religion qui feront appel à lui, et en toutes fonctions, toujours éminentes, qu'il occupera dans l'espace réformé.

5 Si l'on se convertit, prêtre, moine ou théologien, ce peut être par absence de vocation ou/et rejet de la discipline ecclésiastique ou claustrale. Mais il est un autre élément qui fonde en raison cet acte de rupture. J'ai dit la raison de spiritualité, qui ressortit au plus intime d'une "conscience en liberté ». Et qui peut trouver, dans les crises qui ne cessent d'agiter l'Église française, des opportunités d'expression, ou des occasions d'affirmer sa différence. Le jansénisme au XVII ${ }^{e}$ siècle, le port-royalisme au XVIII ${ }^{e}$, furent souvent tenus pour responsables d'avoir ouvert la voie à la Réforme, et l'on ne compte plus les arguments sollicités en ce sens : thème de la grâce, prédestination, communion soumise à l'impératif d'absolue contrition, etc. Le refus des persécutions contre les huguenots et, du même coup, l'exposé des «erreurs » qu'on leur attribuait, a pu permettre, par contrecoup, que soient rassemblés des arguments par ailleurs épars, et qui trouvaient dans le calvinisme un modèle rationalisateur. Que l'abjuration ait pu alors devenir possible, n'impliquait pas qu'elle se soit accomplie sans difficulté. D. Boisson peut en ce sens parler à bon escient de « rupture » - avec la communauté des fidèles, des paroissiens, des confrères.

6 Le pas franchi, restait à se faire admettre par les Églises d'accueil. Genève, principale source d'information analysée par D. Boisson, se distingue par une extrême méfiance à l'égard des convertis. Méfiance redoublée par les réfugiés huguenots ayant trouvé refuge dans la cité de Calvin. Le dernier mot revient au consistoire, qui demande la prestation de fidélité à la foi et à la discipline de l'Église réformée, et impose un délai de quatre ans à tout ecclésiastique converti désireux de devenir ministre. On craint par dessus tout quelque libertinage de l'esprit et une instruction insuffisante, et quelque doute sur la (bonne) foi des impétrants. L'intégration des convertis n'est ainsi pas processus acquis d'avance. Hérétiques, renégats, apostats, aux yeux de l'Église catholique, les convertis sont d'emblée suspects aux yeux des réformés qui les accueillent. Les synodes vont tenter de décourager les projets ecclésiastiques d'avoir charge de ministère. Les récits de la conversion procèdent alors d'une double fonction : ils doivent convaincre les réformés de la radicalité argumentée de leur abjuration, et équilibrer la dénonciation des abus et errements de Rome, par l'adhésion réfléchie à la 
foi nouvelle. Selon D. Boisson, ces récits, malheureusement trop rares, semblent obéir à un même modèle argumentaire, où se retrouvent en effet des confessions personnelles et des considérations théologiques identiques. Charles Drelincourt ou Pierre Du Moulin, ministres, auraient pu inspirer, voire rédiger, certains de ces récits pour leur donner forme persuasive. Dans le cas de Jean de Labadie, sa «Déclaration (...) contenant les raisons » de sa conversion, est assurément trop circonstanciée, et déjà en filigrane dans quelque traité antérieur, pour n'être pas de sa main propre. Les écrits qu'il publiera après 1650 en attesteront. Sans doute retrouve-t-on en d'autres récits comparables, même référence à l'illumination de Paul comme conversion "voulue par Dieu ». Mais Paul va traverser avec une telle insistance l'entière carrière du prédicant Labadie, qu'il s'en faut que l'on en puisse trouver trace ailleurs, et d'égale ferveur, et rigueur. C'est au demeurant pour ce prédicant émérite, l'occasion d'inciter ses anciens coreligionnaires à le rejoindre en pays de Réforme.

7 Il est difficile d'estimer le nombre de convertis agréés ministres au cours des deux siècles étudiés par D. Boisson. Une petite quarantaine, selon les relevés proposés par l'auteur, qui sont de sources sûres, soit environ $10 \%$ de l'échantillon retenu. Charlot d'Argenteuil en est, qui rédige au XviI ${ }^{\mathrm{e}}$ siècle un récit de sa conversion que l'auteur publie en annexe. Et, au même siècle, Jean de Labadie. Au siècle suivant, les cas de Pierre Claris de Florian et d'Yves de Vallone sont présentés, dans l'ouvrage, comme exemplaires d'une conversion réussie. Une même proportion de convertis ne fait pas carrière dans le pastorat, mais, pour ce que les sources peuvent indiquer, dans l'éducation, le journalisme, ou l'artisanat. Au XvII siècle, Nicolas Gueudeville, régulier de la congrégation de Saint-Maur, sera traducteur et journaliste, et J.-B. de Rocoles, bénédictin converti et reconverti, en qualité d'homme de lettres fréquentera Bayle, Basnage, Pictet dans le «salon » de Pierre Fabri, protecteur de cette petite " académie » d'étudiants. Au XviII siècle, La Barre de Beaumarchais, ancien chanoine régulier de Saint-Victor, sera enseignant, quand J.-B. de La Varenne, bénédictin de Saint-Maur, à son tour entrera en journalisme en Provinces-Unies.

Pour l'immense majorité des "prosélytes », les sources archivistiques n'ont pas permis à D. Boisson d'établir l'itinéraire suivi après conversion. Il importe cependant de noter que la conversion, cette "mutation de la foi », peut introduire en l'espace réformé, ce que l'on a pu considérer comme des glissements, ou des transgressions, de doctrine. Labadie n'est pas le seul à connaître des remontrances de l'Église protestante, jusqu'à ce qu'il (se) soit mis en marge de l'institution. Y. de Vallone, après avoir fait preuve d'un calvinisme rigide, tend à se rapprocher des thèses déistes: Bayle, Malebranche, Spinoza - ces références l'y invitent, et le converti en viendra à mettre en cause les dogmes de la Trinité et de la prédestination. Une « religion de la nature » tente ainsi de se définir. Pour La Varenne, note D. Boisson, «la raison et nos penchants naturels doivent être les seuls guides de notre vie». Conversion/subversion? Non, sans doute, mais il faut être attentif à cette mise en doute que ses nouveaux adeptes installent parfois au cœur de la Réforme. M. Veyssière de la Croze, bénédictin de Saint-Maur, abjure en 1696. Son érudition et sa passion de connaissances le conduisent à méditer sur les questions de la tolérance et de l'athéisme, avec sans doute moins d'audace que Bayle, mais dans le même esprit de libre examen.

9 Adversaires résolus de la monarchie absolue, méfiants à l'égard du soulèvement camisard au seuil du XvIII ${ }^{\mathrm{e}}$ siècle, peu concernés par la réorganisation des «Églises du désert ", les convertis partagent pour l'essentiel les positions doctrinales et les 
stratégies politiques des Refuges. Mais D. Boisson montre avec un grand souci de précision que, par delà ces accords de fond, tempérés, on l'a vu, par l'affirmation de tendances qui parurent incompatibles avec l'institution réformée, les convertis ne purent pas réellement et durablement s'intégrer dans leur pays d'accueil. Cela peut expliquer le retour de certains au catholicisme. D'autres, au contraire, vivront leur conversion comme salut personnel, et de cette élection singulière iront de place en place témoigner. Jean de Labadie «le nomade» vaut ici figure centrale d'une conversion poussée aux limites de sa logique, toujours tentée de retourner contre la nouvelle Église les critiques radicales énoncées contre l'allégeance d'hier. Entre ces deux profils extrêmes - retour à Rome/critique de Genève -, la grande majorité des ecclésiastiques venus à la Réforme paraît se fondre dans la masse anonyme des convertis de plein gré, calvinistes en terres de Calvin, anglicans au refuge londonien, toute spécificité gommée par cette adhésion de conformité. L'intérêt fondamental de l'ouvrage de D. Boisson est d'avoir pu identifier une minorité de cas pour qui la conversion demeure un acte essentiel de la conscience, et, comme tel, un élément moteur d'ébranlements plus décisifs dans l'ordre de la spiritualité. 